\title{
Direct Observation of Retrovirus Assembly with FRET, Fluorescence Correlation Spectroscopy, and Single Particle Tracking
}

\author{
Daniel R. Larson*, Yu May Ma ${ }^{\S}$, Volker M. Vogt ${ }^{\S}$, Watt W. Webb* \\ *School of Applied and Engineering Physics, ${ }^{\S}$ Department of Molecular Biology and \\ Genetics, Cornell University, Ithaca, NY 14853
}

The multi-step process of retrovirus assembly is a paradigm for a range of cellular macromolecular interactions, and involves protein-protein, protein-membrane, and protein-nucleic acid interactions. The retrovirus polyprotein Gag directs this assembly process and is the only viral protein required for formation, budding, and release of viruslike particles from the plasma membrane (Gottlinger, 2001; Wills and Craven, 1991). We have developed an approach relying on two-photon fluorescence resonance energy transfer (FRET) and fluorescence correlation spectroscopy (FCS) to directly measure in vivo Gag-Gag interactions and Gag-membrane interactions during the initial stages of assembly. Late stages of assembly, including budding from the plasma membrane, are observed in real time for single viruses using single particle tracking.

The initial steps of retrovirus assembly are still undetermined, and it is unknown if assembly initiates through Gag-Gag interactions in the cytosol, or at the plasma membrane. Both FCS and FRET provide strong evidence for Gag-Gag interactions in the cytoplasm of living cells. Furthermore, FCS measurements of mobility suggest that Gag is present in large complexes in the cytosol, but these complexes are not entirely composed of Gag. Deletion of the NC domain abolishes all Gag-Gag interactions and targeting to the plasma membrane, and deletion of the membrane-binding domain leads to enhanced interactions in the cytosol. Taken together, these results indicate that GagGag interactions occur in the cytosol, are mediated by the NC domain, and are necessary for membrane targeting and budding.

After multimerization and membrane targeting, Gag appears as discrete, subresolution patches on the cell (Figure 1). These patches are thought to be sites of virus assembly and may represent a variety of intermediates ranging from loose aggregates of Gag to nascent virus particles ready to pinch off from the cell. We are able to track these Gag patches on the cell surface for upwards of 3 hours, using a novel algorithm specifically designed for single particle tracking at low signal to noise levels, such as on a cell surface (Thompson et al., 2002). By measuring brightness and location as function of time, we obtain a direct measure of Gag assembly and budding for a single retrovirus.

Finally, the methods developed here also have general applicability to in vivo studies of protein-protein interactions involved in the formation of complex supermolecular structures. 


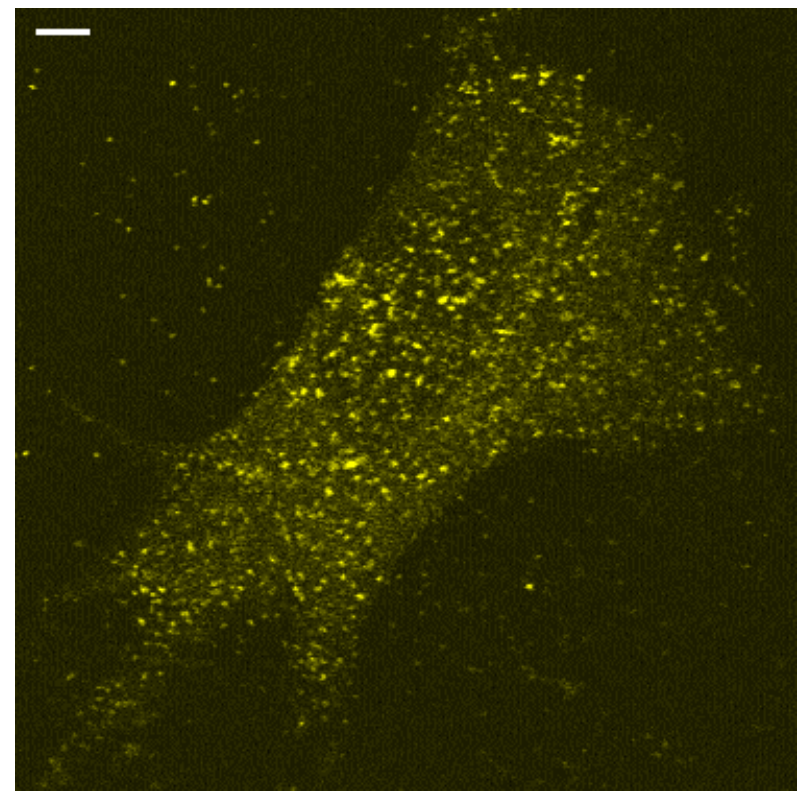

Figure 1: 2-photon laser scanning image of Gag-YFP expressed in DF - 1 cells. The punctate spots are sub-resolution patches of Gag on the surface of the cell in contact with the coverslip. Scale bar $=5 \mu \mathrm{m}$.

Gottlinger, H. G. 2001. The HIV-1 assembly machine. Aids 15 Suppl 5:S13-20.

Thompson, R. E., D. R. Larson and W. W. Webb. 2002. Precise Nanometer Localization Analysis for Individual Fluorescent Probes. Biophys J 82:2775-2783.

Wills, J. W. and R. C. Craven. 1991. Form, function, and use of retroviral gag proteins. Aids 5(6):639-54. 